\title{
Late onset Alzheimer's disease in older people
}

This article was published in the following Dove Press journal:

Clinical Interventions in Aging

9 October 2010

Number of times this article has been viewed

\author{
Ahmet Turan Isik \\ Department of Internal Medicine, \\ Division of Geriatric Medicine, \\ Gulhane School of Medicine, \\ Ankara, Turkey
}

\begin{abstract}
Dementia has become a common diagnosis in aging populations, and the numbers will increase in the forthcoming years. Alzheimer's disease (AD) is the most common form of dementia in the elderly, accounting for $50 \%-56 \%$ of cases at autopsy and in clinical series. Nowadays, the number of people affected by $\mathrm{AD}$ is rapidly increasing, and more than 35 million people worldwide have $\mathrm{AD}$, a condition characterized by deterioration of memory and other cognitive domains, and leading to death 3-9 years after diagnosis. The number of patients with $\mathrm{AD}$, the most common cause of disability in the elderly, is set to rise dramatically. Therefore, it is important for clinicians to recognize early signs and symptoms of dementia and to note potentially modifiable risk factors and early disease markers.
\end{abstract}

Keywords: Alzheimer disease, dementia, elderly

\section{Introduction}

Age is the most important risk factor for $\mathrm{AD}$, with the prevalence rising substantially between the ages of 65 and 85 years. ${ }^{1}$ The incidence of the disease doubles every five years after 65 years of age, with diagnosis of 1275 new cases per year per 100,000 persons older than 65 years, so that $\mathrm{AD}$ affects $30 \%-50 \%$ of all people by the age of 85 years. $^{2}$ Data on centenarians show that AD is not necessarily the outcome of aging, but the odds of receiving a diagnosis of AD after 85 years exceed one in three. ${ }^{3,4}$ Despite its remarkable prevalence among the elderly, AD has been regarded as a specific disease, distinct from normal aging. This view is supported in large part by clinical and pathologic similarities to early-onset, dominantly inherited familial $\mathrm{AD}$, where genetic mutations related to amyloids have been identified. There is much evidence that early onset (sporadic) AD (LOAD) overlaps with normal aging in many clinical and pathologic respects. ${ }^{5}$ Interestingly, early onset AD accounts only for 5\% of total AD cases. The majority of AD patients $(90 \%-95 \%)$ are LOAD, and it usually develops after 65 years of age. ${ }^{6}$

\section{Pathogenesis}

While early onset AD is almost certainly genetically based, there are no specific gene mutations that are associated with inheritance of the disease in LOAD. The expression of the apolipoprotein E (ApoE) 4 allele is one of the risk factors identified for LOAD. ${ }^{7}$ In the central nervous system, ApoE is synthesized by astrocytes, microglia, and, to a lesser extent, by neurons. The role of ApoE in LOAD pathogenesis is not fully elucidated, but it has been suggested that ApoE is important in trafficking of amyloid $\beta$ (A $\beta$ ) peptide. ${ }^{8}$ In addition, apolipoprotein J (clusterin), an amyloid $\beta$-peptide
Correspondence: Ahmet Turan Isik Department of Internal Medicine, Division of Geriatric Medicine, Gulhane School of Medicine, Ankara, Turkey Email:atisik@yahoo.com 
chaperone, TOMM40, a transporter of proteins across the mitochondrial membrane, and Sortillin-related receptor, which functions to partition amyloid precursor protein away from $\beta$-secretase and $\gamma$-secretase, are recently discovered proteins encoded by the risk genes for LOAD. ${ }^{3}$ In addition to nonmodifiable genetic risk factors, potentially modifiable factors, such as hypertension, diabetes mellitus, hyperlipidemia, hyperhomocysteinemia, coronary and peripheral artery diseases, alcohol, smoking, obesity, levels of physical or mental activity, levels of education, and environmental exposures have been investigated to identify risk factors for LOAD., ${ }^{9}$ Furthermore, it has been reported that risk index methods including these risk factors provide a practical, flexible, and objective framework for identifying the optimal combination of measures for identification of high-risk individuals for prevention and early intervention efforts. ${ }^{10}$ Despite the personal and societal burden of LOAD, our understanding of the genetic predisposition to LOAD and the contribution of other risk factors remains limited. More importantly, there are few data to explain the overall risks and benefits of prevention strategies or their impact on risk modification. ${ }^{9}$

$\mathrm{AD}$ is characterized by extensive atrophy of the brain caused by a series neuropathologic changes, including neuronal loss, formation of amyloid plaques, appearance of neurofibrillary tangles, and synaptic loss. ${ }^{11,12}$ Amyloid plaques and neurofibrillary tangles result from an aberration in deposition of the $A \beta$ peptide and the hyperphosphorylated tau protein, respectively, and these depositions lead to neuronal loss and neurotoxicity in the brain affected by AD. ${ }^{13}$ However, these changes in the brain are not found throughout the brain and preferentially affect specific brain areas in a manner that is essentially consistent from patient to patient. ${ }^{14}$ Data obtained by electron microscopy and immunocytochemical and biochemical analysis on synaptic marker proteins in $\mathrm{AD}$ biopsies and autopsies indicate that synaptic loss in the hippocampus and neocortex is an early event and the major structural correlate of cognitive dysfunction. From all cortical areas analyzed, the hippocampus appears to be the most severely affected by the loss of synaptic proteins, while the occipital cortex is affected least. ${ }^{15}$ In addition, it was reported that synaptic loss is currently the best neurobiologic correlate of cognitive deficits in AD. Also, there is evidence that living neurons lose their synapses in AD. Furthermore, synaptic function is impaired in living neurons, as demonstrated by decrements in transcripts related to synaptic vesicle trafficking. ${ }^{16}$

Although new imaging techniques and powerful animal models have helped understanding the time course and the mechanisms of the lesions, the relationship between $A \beta$ accumulation and tau pathology is still badly understood and the mechanism of LOAD continues to be debated. Accumulation of $A \beta$ peptides may be the key event in the pathogenesis of $\mathrm{AD}$. The exact mechanism by which $\mathrm{A} \beta$ peptide deposition induces neurotoxicity is unclear, but it appears that oxidative stress plays an important role. Oxidative stress is extensive in $\mathrm{AD}$, and $\mathrm{A} \beta$ peptides stimulate oxidative stress by both direct and indirect mechanisms. $A \beta$ peptides by themselves may act as enzymes and can bind to mitochondrial proteins, resulting in the generation of free radicals. ${ }^{17,18}$ Furthermore, A $\beta$ peptides generate oxidative stress via neuroinflammation. Considerable evidence has supported the hypothesis that neuroinflammation is associated with $\mathrm{AD}$ pathology. ${ }^{18}$ In addition, in $\mathrm{AD}$, vascular injury and parenchymal inflammation perpetuate the cycle of protein aggregation and oxidation in the brain, and diffuse pathologic changes include cerebral amyloid angiopathy, affecting more than $90 \%$ of patients with $\mathrm{AD}$, capillary abnormalities, disruption of the bloodbrain barrier, and large-vessel channels. ${ }^{3,19}$ It has also been reported that clearance of $\mathrm{A} \beta$ along diseased perivascular channels and through the blood-brain barrier is impeded in $\mathrm{AD}$ atheroma, ${ }^{20}$ and that deregulation of $\mathrm{A} \beta$ transport across the capillary blood-brain barrier is caused by the imbalanced expression of low-density lipoprotein receptor-related proteins and receptors for advanced glycation end products. ${ }^{19,21}$ Furthermore, insulin resistance and hyperinsulinemia are implicated in a number of pathophysiologic processes related to $\mathrm{AD}^{.22,23}$ It has been demonstrated that reduced brain insulin signaling is associated with increased tau phosphorylation and $A \beta$ levels in a streptozotocin-induced model of diabetes mellitus, ${ }^{24,25}$ and also that insulin promotes the release of intracellular $A \beta$ in neuronal cultures and accelerates $A \beta$ trafficking to the plasma membrane. Similarly, intravenous insulin infusion also raises plasma $A \beta 42$ levels in patients with $\mathrm{AD}$ but not in normal adults, an effect that is exaggerated in patients with $\mathrm{AD}$ and a higher body mass index. In addition, impaired insulin or insulin-like growth factor-1 signaling can result in hyperphosphorylation of tau, which can cause cell death mediated by apoptosis, mitochondrial dysfunction, or necrosis, and promote oxidative stress, which contributes to the neurodegeneration cascade, and leads to dementiaassociated behavioral and cognitive deficits. For this reason, it seems that insulin resistance causes tau phosphorylation, neurofibrillary tangle formation, and increased beta amyloid aggregation in LOAD. ${ }^{22,23-25}$ Therefore, we think that the insulin resistance should be taken into account, both in explaining the pathophysiologic mechanisms and the managing of the 
LOAD. In short, the current pathophysiologic approach to LOAD is based on a number of common mechanisms of neurodegeneration, including accumulation of abnormal proteins, mitochondrial dysfunction, and oxidative stress, impaired insulin signaling, calcium homeostasis dysregulation, early synaptic disconnection, and late apoptotic cell death. Aging itself is associated with mild cognitive deterioration, probably due to subtle multifactorial changes resulting in a global decrease of functional brain reserve. .,22,26 $^{2}$

\section{Symptoms}

AD can affect different people in different ways, but the most common symptom pattern begins with gradually worsening difficulty remembering new information. In the early stages, the most commonly recognized symptom is inability to acquire new memories, such as difficulty in recalling recently observed facts. ${ }^{27}$ Cognitive profiles of normal aging emphasize a decline in skills, including learning efficiency, working memory, and psychomotor speed. Although memory impairment is the earliest cognitive change in $\mathrm{AD}$, distinguishing early disease from normal aging can be difficult, and making a decision as to whether a memory complaint is associated with the normal aging process or is a precursor sign for dementia, is difficult for the doctor. ${ }^{28-30}$ Therefore, the earliest observable symptoms are often mistakenly thought to be age-related concerns, or manifestations of stress. ${ }^{31}$ When $\mathrm{AD}$ is suspected, the diagnosis is usually confirmed by behavioral assessment and cognitive tests, often followed by a brain scan if possible. ${ }^{31}$ As the damage spreads, individuals also experience confusion, disorganized thinking, impaired judgment, trouble expressing themselves, and disorientation. The following are warning signs of Alzheimer's disease:

- Memory loss that disrupts daily life

- Challenges in planning or solving problems

- Difficulty completing familiar tasks at home, at work, or at leisure

- Confusion in time or place

- Trouble understanding visual images and spatial relationships

- New problems with words in speaking or writing

- Misplacing things and losing the ability to retrace steps

- Decreased or poor judgment

- Withdrawal from work or social activities

- Changes in mood and personality. ${ }^{27}$

As the disease advances, symptoms include confusion, irritability, and aggression, mood swings, language breakdown, long-term memory loss, and the general withdrawal of the sufferer as their senses decline. ${ }^{31}$ As the disease progresses, cognitive impairment becomes profound and daily functioning skills decline. Although typically thought of as indicative of late-stage disease, behavioral symptoms can appear early in the course of the disease, well before clinical diagnosis. These symptoms can include social withdrawal, depression, paranoia, and mood changes. As the disease advances, symptoms such as anxiety, irritability, and agitation become more pronounced. ${ }^{32}$ Behavioral symptoms are also a major source of stress for the caregiver. Behavioral disturbances have been shown to be a strong predictor of caregiver burden ${ }^{8}$ and are associated with increased financial hardship for the caregiver. ${ }^{33}$ Physical functions are gradually lost, ultimately leading to death. Individual prognosis is difficult to assess, because the duration of the disease varies. AD develops for an indeterminate period of time before becoming fully apparent, and it can progress undiagnosed for years. The mean life expectancy following diagnosis is approximately 7 years. ${ }^{34}$ Fewer than $3 \%$ of individuals live more than 14 years after diagnosis. ${ }^{35}$

\section{Diagnosis}

There is an increasing interest in the identification of patients in the earliest stage of $\mathrm{AD}$, prior to clinical manifestation of dementia, in order to provide effective early intervention that aims at delaying significant impairment. A definitive diagnosis of $\mathrm{AD}$ requires a detailed post-mortem microscopic examination of the brain. But nowadays, AD can be diagnosed with more than $95 \%$ accuracy in living patients by using a combination of tools. These include taking a careful history from patients and their families, and assessing cognitive function by neuropsychologic tests. Other causes of dementia must be ruled out, such as low thyroid function, vitamin deficiencies, infections, cancer, and depression. It is also crucial to differentiate $\mathrm{AD}$ from other neurodegenerative dementias. ${ }^{36}$

The National Institute of Neurological and Communicative Disorders and Stroke and the Alzheimer's Disease and Related Disorders Association (NINCDS-ADRDA) and the Diagnostic and Statistical Manual of Mental Disorders, Fourth Edition, Text Revision (DSM-IV-TR) criteria for AD are the prevailing diagnostic standards in research. However, they have now fallen behind the unprecedented growth of scientific knowledge. Moreover, NINCDS-ADRDA was reported in 1984 and the subsequent DSM-IV-TR in 2000. For this reason, to improve the specificity for diagnosis of $A D$, the criteria were revised by Dubois to offer a new diagnostic approach including genetic testing, molecular imaging, and body fluid biomarkers. ${ }^{37}$ Furthermore, draft reports presented 
at the International Conference on Alzheimer's Disease in 2010 will form the basis of new diagnostic criteria for mild cognitive impairment and AD.

\section{Treatment}

There was no effective therapy for AD before the approval of the cholinesterase inhibitors and memantine. These agents are associated with detectable symptomatic improvement, and have a modest effect on the progression of $\mathrm{AD}$ from mild cognitive impairment to disabling dementia and death. ${ }^{38}$ Medicines currently prescribed for AD fall into three groups, ie, inhibitors of acetylcholinesterase (according to the cholinergic hypothesis of $\mathrm{AD}$, memory impairments result from death of cholinergic neurons in the basal forebrain), an antagonist of a receptor for the neurotransmitter glutamate, and drugs from the psychiatric toolbox to control depression and behavioral abnormalities. ${ }^{36}$

The clinical development of new agents for symptomatic and disease-modifying treatment of AD has resulted in both promise and disappointment. Despite the fact that no new compound for the treatment of $\mathrm{AD}$ has been introduced since the approval of memantine in 2002, the variety of drug targets and mechanisms of action, and the total number of compounds under investigation make it highly likely that important new pharmacotherapeutic options will become available for the treatment of AD over the next decade. Moreover, research into the underlying etiology and pathophysiology of $\mathrm{AD}$ is likely to facilitate identification of additional targets for future drug development. ${ }^{38}$ In addition, stem cell therapy for AD might be used to replace destroyed neurons, but AD poses particular challenges in this regard because it affects diverse types of neurons in different brain regions. ${ }^{36}$ However, our experience has demonstrated that mesenchymal stem cell therapy might provide neuronal replacement and improved cognitive function in streptozotocin-induced neurodegeneration in rats, but adjunctive therapies with mesenchymal stem cells in this type of neurodegeneration need to be tried. ${ }^{39}$ However, the development of bone marrow mesenchymal stem cell therapy for the replacement of cells and tissues lost due to neurodegeneration in AD is still in the early stages, and further studies will be needed before it can be tested in humans. Nonetheless, these improving effects of mesenchymal stem cells give us hope for the future.

\section{Prevention}

Nowadays, there is no definitive evidence to support any particular measure being effective in prevention of $\mathrm{AD}$.
Global studies of measures to prevent or delay the onset of $\mathrm{AD}$ have often produced inconsistent results. However, epidemiologic studies have proposed relationships between certain modifiable factors, such as diet, cardiovascular risk, pharmaceutical products, or intellectual activities, among others, and a population's likelihood of developing AD. Only further research, including clinical trials, will reveal whether these factors can help to prevent AD. ${ }^{40}$ In addition, at the International Conference on Alzheimer's Disease in 2010, it was also reported that moderate to heavy physical activity is associated with a reduced risk of dementia, with up to two decades of follow-up. ${ }^{41}$

\section{Conclusion}

In summary, $\mathrm{AD}$ is increasingly being diagnosed as one of the most important medical problems in the elderly, and the management of elderly patients with $\mathrm{AD}$ is complex. A comprehensive approach is required that focuses on both the patient and caregiver. Despite all developments, our treatment options for prevention and treatment of the cognitive, behavioral, and psychologic symptoms of $\mathrm{AD}$ are still lacking. Therefore, it is important for clinicians to recognize early signs and symptoms of $\mathrm{AD}$ and to determine potentially modifiable risk factors.

\section{Disclosure}

The author reports no conflicts of interest in this work.

\section{References}

1. Sloane PD, Zimmerman S, Suchindran C, et al. The public health impact of Alzheimer's disease, 2000-2050: Potential implication of treatment advances. Annu Rev Public Health. 2002;23:213-231.

2. Hirtz D, Thurman DJ, Gwinn-Hardy K, et al. How common are the "common" neurologic disorders? Neurology. 2007;68:326-337.

3. Querfurth HW, LaFerla FM. Mechanisms of disease: Alzheimer's disease. N Engl J Med. 2010;362:329-344.

4. den Dunnen WF, Brouwer WH, Bijlard E, et al. No disease in the brain of a 115-year-old woman. Neurobiol Aging. 2008;29:1127-1132.

5. Drachman DA. Aging of the brain, entropy, and Alzheimer disease. Neurology. 2006;67:1340-1352.

6. Harman D. Alzheimer's disease pathogenesis: Role of aging. Ann NY Acad Sci. 2006;1067:454-460.

7. Schellenberg GD. Genetic dissection of Alzheimer's disease, a heterogeneous disorder. Proc Natl Acad Sci U S A. 1995;92: $8552-8559$.

8. Holtzman DM. Role of ApoE/A $\beta$ interactions in the pathogenesis of Alzheimer's disease and cerebral amyloid angiopathy. J Mol Neurosci. 2001; $17: 147-155$.

9. Patterson C, Feightner JW, Garcia A, et al. Diagnosis and treatment of dementia: 1. Risk assessment and primary prevention of Alzheimer disease. CMAJ. 2008;178:548-556.

10. Barnes DE, Covinsky KE, Whitmer RA, et al. Commentary on "Developing a national strategy to prevent dementia: Leon Thal Symposium 2009." Dementia risk indices: A framework for identifying individuals with a high dementia risk. Alzheimers Dement. 2010;6: 138-141. 
11. Bussiere T, Giannakopoulos P, Bouras C, et al. Progressive degeneration of nonphosphorylated neurofilament protein-enriched pyramidal neurons predict cognitive impairment in Alzheimer's disease: Stereologic analysis of prefrontal cortex area. J Comp Neurol. 2003;463: 281-302.

12. Garry LW. Neuropathologic changes in Alzheimer's disease: Potential target for treatment. J Clin Psychiatry. 2006;67 Suppl 3:3-7.

13. Cotman CW, Su JH. Mechanisms of neuronal death in Alzheimer's disease. Brain Pathol. 1996;6:493-506.

14. Braak H, Braak E. Neuropathologic staging of Alzheimer-related changes. Acta Neuropathol (Berl). 1991;82:239-259.

15. Arendt T. Synaptic degeneration in Alzheimer's disease. Acta Neuropathol. 2009;118:167-179.

16. Coleman PD, Yao PJ. Synaptic slaughter in Alzheimer's disease. Neurobiol Aging. 2003;24:1023-1027.

17. Lustbader JW, Cirilli M, Lin C. ABAD directly links Abeta to mitochondrial toxicity in Alzheimer's disease. Science. 2004;304:448-452.

18. Newman M, Musgrave FI, Lardelli M. Alzheimer disease: Amyloidogenesis, the presenilins and animal models. Biochimica Biophysica Acta. 2007;1772:285-297.

19. Weller RO, Boche D, Nicoll JAR. Microvasculature changes and cerebral amyloid angiopathy in Alzheimer's disease and their potential impact on therapy. Acta Neuropathol. 2009;118:87-102.

20. Price JM, Chi X, Hellermann G, Sutton ET. Physiological levels of beta-amyloid induce cerebral vessel dysfunction and reduce endothelial nitric oxide production. Neurol Res. 2001;23:506-512.

21. Deane R, Zlokovic BV. Role of the blood-brain barrier in the pathogenesis of Alzheimer's disease. Curr Alzheimer Res. 2007;4:191-197.

22. Isik AT, Bozoglu E. Insulin resistance in Alzheimer's disease. In: Aucoin L, Prideux T, editors. Handbook of Type 1 Diabetes Mellitus: Etiology, Diagnosis, and Treatment. Hauppauge, NY: Nova Science; 2010.

23. Isik AT, Celik T, Ulusoy G, et al. Curcumin ameliorates impaired insulin/ IGF signaling and memory deficit in streptozotocin treated rat model. Age. 2009;31:39-49.

24. Jolivalt CG, Lee CA, Beiswenger KK, et al. Defective insulin signaling pathway and increased glycogen synthase kinase-3 activity in the brain of diabetic mice: Parallels with Alzheimer's disease and correction by insulin. J Neurosci Res. 2008;86:3265-3274.

25. de la Monte SM, Wands JR. Review of insulin and insulin-like growth factor expression, signaling, and malfunction in the central nervous system: Relevance to Alzheimer's disease. J Alzheimers Dis. 2005;7:45-61.
26. Popescu BO, Toescu EC, Popescu LM, et al. Blood-brain barrier alterations in ageing and dementia. J Neurol Sci. 2009;283:99-106.

27. Alzheimer's Association. 2010 Alzheimer's disease facts and figures. Alzheimers Dement. 2010;6:158-194.

28. Salthouse TA. Memory aging from 18 to 80. Alzheimer Dis Assoc Disord. 2003;17:162-167.

29. Treitz FH, Heyder K, Daum I. Differential course of executive control changes during normal aging. Neuropsychol Dev Cogn B Aging Neuropsychol Cogn. 2007;14:370-393.

30. Isik AT. Approach the elderly patient with forgetfulness. Journal of Geriatrics and Geriatric Neuropsychiatry. 2009;2:33-38.

31. Waldemar G, Dubois B, Emre M, et al. Recommendations for the diagnosis and management of Alzheimer's disease and other disorders associated with dementia: EFNS guideline. Eur J Neurol. 2007;14(1):e1-e26.

32. Jost BC, Grossberg GT. The evolution of psychiatric symptoms in Alzheimer's disease: A natural history study. JAm Geriatr Soc. 1996;44: 1078-1081.

33. Beier MT. Treatment strategies for the behavioral symptoms of Alzheimer's disease: Focus on early pharmacologic intervention. Pharmacotherapy. 2007;27(3):399-411.

34. National Institute on Aging. Understanding stages and symptoms of Alzheimer's disease. 2007. Available at: http://www.nia.nih.gov/ alzheimers/publications/stages.htm. Accessed 2008 Feb 21.

35. Mölsä PK, Marttila RJ, Rinne UK. Long-term survival and predictors of mortality in Alzheimer's disease and multi-infarct dementia. Acto Neurol Scand. 1995;91:159-164.

36. Mucke L. Alzheimer disease. Nature. 2009;461:895-897.

37. Dubois B, Feldman F, Jacova C, et al. Research criteria for the diagnosis of Alzheimer's disease: Revising the NINCDS-ADRDA criteria. Lancet Neurol. 2007;6:734-746.

38. Sabbagh NM. Drug development for Alzheimer's disease: Where are we now and where are we headed? Am J Geriatr Pharmacother. 2009; 7:167-185.

39. Isik AT, Celik T, Ural AU, Tosun M, et al. Does the mesenchymal stem cell therapy improve the streptozotocin induced neurodegeneration in rats? Alzheimers Dement. 2010;6 Suppl 1:569.

40. Szekely CA, Breitner JC, Zandi PP. Prevention of Alzheimer's disease. Int Rev Psychiatry. 2007;19:693-706.

41. Tan ZS, Beiser AS, Au R, et al. Physical activity and risk of dementia: The Framingam study. Alzheimers Dement. 2010;6 Suppl 1:S68.
Clinical Interventions in Aging

\section{Publish your work in this journal}

Clinical Interventions in Aging is an international, peer-reviewed journal focusing on evidence-based reports on the value or lack thereof of treatments intended to prevent or delay the onset of maladaptive correlates of aging in human beings. This journal is indexed on PubMed Central, MedLine, the American Chemical Society's 'Chemical Abstracts

\section{Dovepress}

Service' (CAS), Scopus and the Elsevier Bibliographic databases. The manuscript management system is completely online and includes a very quick and fair peer-review system, which is all easy to use. Visit http://www.dovepress.com/testimonials.php to read real quotes from published authors. 